\title{
A STUDY OF Be STARS IN CLUSTERS
}

\author{
R. SCHILD \\ Center for Astrophysics, Cambridge, Mass., U.S.A. \\ and \\ W. ROMANISHIN \\ Dept. of Astronomy, University of Arizona, Tucson, Ariz., U.S.A.
}

\begin{abstract}
Calibrated spectrograms at $\mathrm{H} \alpha$ of 566 stars in 29 young galactic clusters led to the detection of $41 \mathrm{Be}$ stars in clusters. Using cluster membership we have inferred ages and intrinsic $(B-V)$ colors of $\mathrm{Be}$ stars to permit a discussion of their evolutionary states.

Rotating stars can become Be stars in their early hydrogen burning evolution away from the main sequence. Both the fraction of stars showing hydrogen emission and the strength of emission appear to vary little during the first $80 \%$ of post main sequence evolution. However, at the onset of gravitational core contraction, both the fraction of stars showing emission and their mean emission strength undergo a fourfold increase. Many stars in the core contraction phase develop an intrinsic $(B-V)$ excess of 0.15 mag. due to the $\mathrm{H}^{-}$free-bound continuum radiation. Because of the great strength of $\mathrm{H} \alpha$ emission and the short time duration of the effect, the extreme Be stars would be excellent probes for studies of spiral structure and would also serve as probes for studies of ages and distances of extragalactic systems.

Analysis of the corrected colors of the Be stars in clusters suggests that the Lucy and Solomon (1970) mechanism for reduction of effective surface gravity by ultraviolet resonance line scattering is probably important for the hotter Be stars. However, a discrepancy exists for the cooler stars between the predicted and observed colors.
\end{abstract}

\section{References}

Lucy, L. and Solomon, P.: 1970, Astrophys. J. 159, 879.

\section{DISCUSSION}

Conti: I was particularly struck by the absence of any strong correlation between $\mathrm{H} \alpha$ emission strength and $\tau$, the 'core-contraction' parameter. There were only a few stars, just at $\tau=1$, with strong $H \alpha$. Could this just be those earliest B stars with wind enhanced envelopes? Or possibly 'mass exchanging' binaries with enhanced emission, or slightly modified $M_{v}$ and $B-V$ parameters?

Schild: I worried about this, but I can't imagine why the binaries would all have $\tau$ between 0.8 and 1.0, although perhaps there is a reason.

Plavec: There may be a reason. Recently, Ulrich and Burger at UCLA studied the accretion of the material on a binary component. When the rate of mass transfer gets higher the accreting star becomes overluminous and is displaced away from the main sequence. What you would expect in these systems, if they are really exchanging material, is that the observed Be star, when it is surrounded by this material, will also be displaced away from the main sequence into the region where the stars normally are when they are leaving the main sequence. So that is one possible explanation.

Young: Does your study suggest that there is a significant difference in the frequency and appearance of Be stars among early B stars that are in galactic clusters, vs those that are field stars?

Schild: That is a difficult question to answer, because relatively few complete surveys of this kind have been made for field stars and you have all the problems of systematic effects such as the intrinsic $(B-V)$ and $(U-B)$ color excesses in the field stars which are basically avoided by going to the clusters. Thus $I$ cannot give any kind of reasonable answer to your question.

Young: In the field of galactic clusters there are a number of workers who have shown that there is a remarkable inverse correlation between high frequency of binaries in clusters and low rotation. Does your sample overlap theirs so that you can say something about this? 
Schild: Unfortunately I cannot. Helmut Abt and collaborators have observed primarily A-type stars and I am an observer of B-type stars, so he tended to select clusters by different criteria. I cut off at about the temperature where Abt starts, and there is essentially no overlap.

Cowley: Recent work by Crampton at the Dominion Astrophysical Observatory has shown that the correlation that $\mathrm{Abt}$ found between stellar rotation and binary frequency in clusters may not be supported by more recent observations.

Henize: Since my southern $\mathrm{H} \alpha$ survey probably includes the most uniform data available on the field stars, let me comment that, even though my survey data to a fixed magnitude limit show a peak frequency at HD class B8, the emission frequency per hundred stars of the same spectral class still shows a clear peak at B2. I find that one star in five shows emission at B2 while only one star in 30 shows emission at B8,9. This is quite different from the statistics Schild finds in his cluster data.

Slettebak: In your earlier paper in which you define extreme Be stars, you define them also in terms of sharp helium lines, which implies small rotation. Do you have any information from this sample about line widths?

Schild: No. These spectrograms are of relatively low quality. They were $\mathrm{H} \alpha$ spectrograms taken with an image tube and were widened to only $0.2 \mathrm{~mm}$. I did not try to measure line widths. Dr Slettebak is fully aware that I had the idea of two kinds of Be stars back in 1966. There are now a number of criteria which I have used to identify extreme Be stars: I made infrared observations, continuum scans, and spectroscopic observations, and I am still having the greatest difficulty in really showing that there are two separate, different kinds of Be stars (the core-contraction stars and the normal post-main sequence stars). It is very difficult to tie together all these different kinds of observations in one consistent definition which applies to all Be stars over the temperature range $10-30000 \mathrm{~K}$.

Henize: Do your data or your theoretical concepts lead to a prediction of how emission-line strengths are distributed for a particular spectral class? I ask this because my survey data show that weak emission lines are conspicuously infrequent among the B0-B2 stars, thus suggesting an all-on or all-off mechanism for triggering the emission.

Schild: I have no information. 